\section{Commentary: Hidden treasure: Cultivating pearls from a sea of information}

\author{
Joseph S. Coselli, MD
}

Total transverse aortic arch replacement is in many ways one of the last frontiers of open cardiovascular surgery. The aortic arch and its branching arteries are complex, necessitating a nuanced and adaptable approach that is tailored to the individual needs of patients; the flexibility needed for successful repair tends to cautiously balance the use of open techniques and emerging endovascular techniques. As surgeons standing on the precipice of change, we must truly understand the breadth of our vast technical armamentarium to guide patient-specific repair. Even for authors of books solely focused on aortic arch repair, ${ }^{1}$ navigating the abundance of contemporary material is an ongoing challenge as the literature grows exponentially. Ultimately, all surgeons need to understand what works best for them and their team to make reasonably safe decisions and avoid drastic scenarios.

Although much of the controversy of aortic arch repair has centered on technical issues such as hypothermic temperature, cannulation sites and methods, and an indication-guided extent of repair, less attention has been paid to the various methods by which the entire aortic arch and its supra-aortic branching arteries can be replaced with graft. In this issue of JTCVS Techniques, Gambardella and Girardi ${ }^{2}$ present an overview of total arch replacement with the goal of providing the reader with clear direction regarding how to best select the method of replacement

\footnotetext{
From the Division of Cardiothoracic Surgery, Michael E. DeBakey Department of Surgery, Baylor College of Medicine; Section of Adult Cardiac Surgery, Department of Cardiovascular Surgery, Texas Heart Institute; and CHI St Luke's Health-Baylor St Luke's Medical Center, Houston, Tex.

Disclosures: The author reported no conflicts of interest.

The Journal policy requires editors and reviewers to disclose conflicts of interest and to decline handling or reviewing manuscripts for which they may have a conflict of interest. The editors and reviewers of this article have no conflicts of interest.

Received for publication May 26, 2021; revisions received May 26, 2021; accepted for publication June 1, 2021; available ahead of print June 6, 2021.

Address for reprints: Joseph S. Coselli, MD, Baylor College of Medicine, One Baylor Plaza, BCM 390, Houston, TX 77030-3411 (E-mail: jcoselli@bcm.edu).

JTCVS Techniques 2021;10:14-5

2666-2507

Copyright (c) 2021 The Author(s). Published by Elsevier Inc. on behalf of The American Association for Thoracic Surgery. This is an open access article under the CC BY-NC-ND license (http://creativecommons.org/licenses/by-nc-nd/4.0/).

https://doi.org/10.1016/j.xjtc.2021.06.007
}

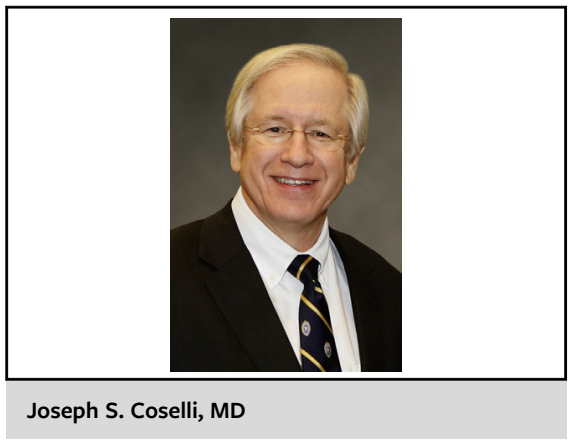

\begin{abstract}
CENTRAL MESSAGE
In total arch replacement, the stakes are high. Surgeons can benefit from collective experience distilled into easily digested pearls of wisdom.
\end{abstract}

for a given patient. The authors do more than provide a vast spectrum of replacement configurations-they also address important questions such as why you would prioritize one strategy of reattachment over another. For example, the authors highlight crucial strategies such as the en bloc technique, which was first proposed more than 5 decades ago by Bloodwell, Hallman, and Cooley to directly reattach the arch vessels as a patch or island. ${ }^{3}$ This approach is best reserved for older patients with degenerative aneurysms, rather than younger patients with heritable thoracic aortic disease; older patients will benefit from this simpler reconstruction with fewer operative demands and do not have to pay the price of late aneurysmal patch degeneration, which is common in younger patients. However, the authors astutely note that this paradigm does not apply if the distal aspect of the proposed patch anastomosis is too deep in the chest to be easily performed, potentially resulting in the loss of any benefit if uncontrolled bleeding occurs. In contrast, the authors promote graft replacement of supra-aortic branching arteries in young patients with heritable thoracic aortic disease to reduce native residual aortic tissue. Further, the authors provide a wealth of instruction for handling anatomic variants of branching arteries, which are not uncommon.

The authors progress from one of the oldest approaches to total aortic replacement to one of the most recent: the frozen elephant trunk approach. ${ }^{4}$ The frozen elephant trunk builds on the legacy of the classic elephant trunk ${ }^{5}$-in which a short section of graft extends from the distal 
anastomosis-by replacing this section of graft with a stentgraft. The pearls here regard the complexities of "homemade" strategies for frozen elephant trunk repairs that developed because of a lack of device access in the United States, as well as the benefits of manufactured, one-piece devices available outside the United States. The ability to extend repair to the proximal portion of the descending thoracic aorta is a clear advantage of the frozen elephant trunk. The authors emphasize that aortic disease does not discretely stop at the anatomical edge of the transverse aortic arch but rather extends (or has the propensity to subsequently extend) into the distal aorta.

In total arch replacement, the stakes are high. Many patients are reoperative, which means that any failure related to the method of repair could necessitate a third sternotomy, which can significantly increase operative risk. Choosing the best approach, one that meets both today's and tomorrow's needs, requires a deep understanding of common scenarios of aortic disease progression. We all know that a pearl is worthless if it is trapped in its shell-Gambardella and Girardi have done the hard work of synthesizing decades of experience into easily digested pearls of wisdom.

\section{References}

1. Coselli JS, LeMaire SA. Aortic Arch Surgery: Principles, Strategies and Outcomes. West Sussex, UK: Wiley-Blackwell; 2008.

2. Gambardella I, Girardi LN. Total arch replacement: technical pearls. J Thorac Cardiovasc Surg Tech. 2021;10:8-13.

3. Bloodwell RD, Hallman GL, Cooley DA. Total replacement of the aortic arch and the "subclavian steal" phenomenon. Ann Thorac Surg. 1968;5:236-45.

4. Shrestha M, Beckmann E, Krueger H, Fleissner F, Kaufeld T, Koigeldiyev N, et al The elephant trunk is freezing: the Hannover experience. J Thorac Cardiovasc Surg. 2015;149:1286-93.

5. Borst HG, Walterbusch G, Schaps D. Extensive aortic replacement using "elephant trunk" prosthesis. Thorac Cardiovasc Surg. 1983;31:37-40. 